\title{
Thrombotic microangiopathy following haematopoietic stem cell transplant
}

\author{
Eleanor G. Seaby ${ }^{1} \cdot$ Rodney D. Gilbert ${ }^{2}$
}

Received: 17 February 2017 /Revised: 23 August 2017 / Accepted: 1 September 2017 /Published online: 9 October 2017

(C) The Author(s) 2017. This article is an open access publication

\begin{abstract}
Thrombotic microangiopathy is a potentially lethal complication of haematopoietic stem cell (bone marrow) transplantation. The pathophysiology is incompletely understood, although endothelial damage appears to be central. Platelet activation, neutrophil extracellular traps and complement activation appear to play key roles. Diagnosis may be difficult and universally accepted diagnostic criteria are not available. Treatment remains controversial. In some cases, withdrawal of calcineurin inhibitors is adequate. Rituximab and defibrotide also appear to have been used successfully. In severe cases, complement inhibitors such as eculizumab may play a valuable role. Further research is required to define the pathophysiology and determine both robust diagnostic criteria and the optimal treatment.
\end{abstract}

Keywords Thrombotic microangiopathy $\cdot$ Hematopoietic stem cell transplant · Acute kidney injury · Complement . Neutrophil extracellular traps

\section{Introduction}

The thrombotic microangiopathies (TMAs) are a group of disorders defined by intravascular activation and sequestration of platelets, resulting in the accumulation of platelet- and

Rodney D. Gilbert

Rodney.Gilbert@uhs.nhs.uk

1 Human Genetics and Genomics Department, University of Southampton, Southampton, UK

2 Southampton Children's Hospital and Faculty of Medicine, University of Southampton, Tremona Road, Southampton SO16 6YD, UK fibrin-rich micro-thrombi that occlude and disrupt the microcirculation, fragmenting erythrocytes as they flow through the vessel lumen [1]. Disorders classified as TMAs include, but are not limited to: thrombotic thrombocytopaenic purpura (TTP); haemolytic uraemic syndrome (HUS); haemolysis, elevated liver enzymes and low platelets (HELLP) syndrome; and transplant-associated thrombotic microangiopathy (TATMA) [2]. Although these disorders share common features, with advances in genomics and histo-biology we are beginning to understand the aetiological differences that drive a final common pathway of endothelial damage that results in TMA and target organ damage [2,3].

The first descriptions of TA-TMA were published in the early 1980 s with observations of endothelial damage complicating bone marrow transplant in those receiving cyclosporine A for graft versus host disease (GVHD) prophylaxis $[4,5]$. Since then, our understanding of what drives TA-TMA and exactly where it lies on the spectrum of TMA has been disputed and partially resolved. Features of TA-TMA include microangiopathic, Coombs-negative haemolytic anaemia and thrombocytopaenia, high serum lactate dehydrogenase (LDH) levels, decreased or undetectable haptoglobin levels, schistocytosis on peripheral blood smear, target organ damage and transfusion requirements. It is therefore understandable how the overlapping features of TA-TMA with most notably TTP and HUS have led to scientific debate about whether TATMA is merely a variant of TTP or HUS, or a completely distinct clinical entity $[3,6]$. There is now convincing evidence that TA-TMA is distinct from TTP and may represent a type of HUS [2, 3], as demonstrated by its response to eculizumab therapy [7, 8]. Classical TTP results from a deficiency of a disintegrin and metalloprotease with a thrombospondin type 1 motif, member 13 (ADAMTS13) activity, leading to an accumulation of multimeric, prothrombotic von Willebrand factor (vWF) that drives 
intravascular thrombosis by the agglutination of circulating platelets under high shear stress [9]. In most patients with TA-TMA, ADAMTS13 activity is greater than 5 to $10 \%$, metalloprotease activity is normal [3] and autoantibody inhibitors of vWF cleaving protease are undetectable, which is distinct from classical TTP. Furthermore, TTP is typically characterised by vWF in non-inflammatory, platelet-rich "white" thrombi [7], unlike the inflammatory, fibrin-rich "red" thrombi with C5b-9 deposition, caused by aberrant alternative pathway mechanisms of complement activation more commonly seen in HUS and TA-TMA [3, 7, 10, 11].

\section{Incidence}

Reported incidence rates of TA-TMA vary widely, from 0.5 to $76 \%[10,12-14]$, although more recently, sources aggregate the incidence between 12 and $35 \%[6,15,16]$. Some of the challenge of estimating incidence lies in the extremely variable clinical presentation, ranging from asymptomatic anaemia, thrombocytopaenia or mild acute kidney injury to fulminating multisystem disease [6]. Different centres use different criteria for diagnosing TATMA (Table 1) and there may be difficulty distinguishing clinical features caused by other haematopoietic stem cell transplant (HSCT)-related complications, e.g. infection, GVHD and cyclosporine toxicity [14].

Table 1 Comparison of current diagnostic criteria for transplantassociated thrombotic microangiopathy (TA-TMA). NB: for Jodele's criteria, if 1,2, 3 are present, consider a diagnosis of TA-TMA and

\section{Aetiology and risk factors}

Accurately discerning the aetiology of TA-TMA is hindered by the definition of TA-TMA. What is clear is that something intrinsic to HSCT provokes TA-TMA, and unpicking these triggers is important to understanding the pathogenesis of the disease. Several theories have been proposed to explain TA-TMA. Risk factors are likely to include intrinsic donor and recipient properties, in addition to extrinsic modifiers, such as conditioning regimens [19], viral infections, immunosuppressive therapies, especially combined sirolimus and cyclosporine regimens [20], and GVHD, all of which may damage endothelium and lead to a cascade of aberrant complement activation and TMA [7].

\section{Intrinsic factors}

The most notable intrinsic factors associated with TA-TMA include female sex, advancing age and genetic predisposition $[14,21]$. Although some reports have concluded that female gender is not a risk factor for TA-TMA [13, 22], other studies have found that females are statistically more likely to develop the disease than their male counterparts $[14,23]$. The pathophysiology of this effect remains speculative; hypotheses include hormonal differences between men and women, potentially influenced by oral contraception use and pregnancy. Of note, HSCT from female donors is not an independent risk factor for TA-TMA, implicating the host environment as the source of increased risk [23].

monitor very closely. Otherwise, 2 and 4 indicate features associated with poor outcome; therefore, consider therapeutic intervention $[6,12,17,18]$

$\begin{array}{llll}\text { Leukaemia Net } & \text { Blood and Marrow } & \text { Probably TMA [18] } & \text { Jodele's proposed criteria [6] } \\ \text { International Working } & \text { Transplant Clinical Trials } & & \\ \text { Group [17] } & \text { Network [12] } & \end{array}$

\begin{tabular}{|c|c|c|c|c|}
\hline Platelet count & $\begin{array}{l}<50,000 / \mathrm{mm}^{3} \text { or }<50 \% \\
\text { of baseline }\end{array}$ & N/A & $\begin{array}{l}<50,000 / \mathrm{mm}^{3} \text { or }<50 \% \text { of } \\
\text { baseline }\end{array}$ & $<50,000 / \mathrm{mm}^{3}$ or $<50 \%$ of baseline \\
\hline Schistocytes & $>4 \%$ & $>2$ per high power field & $\begin{array}{l}\text { Present in peripheral blood or } \\
\text { microangiopathy on tissue } \\
\text { specimen }\end{array}$ & $\begin{array}{l}\text { Present in peripheral blood or } \\
\text { microangiopathy on tissue specimen }\end{array}$ \\
\hline LDH & Increased & Increased & Increased & Increased \\
\hline Haptoglobin & Decreased & N/A & $\begin{array}{l}\text { De novo anaemia with } \\
\text { haptoglobin below lower } \\
\text { limit of normal }\end{array}$ & N/A \\
\hline Transfusions & Increased & N/A & $\begin{array}{l}\text { Anaemia requiring transfusion } \\
\text { support }\end{array}$ & $\begin{array}{l}\mathrm{Hb} \text { below lower limit of normal for age or } \\
\text { transfusion support }\end{array}$ \\
\hline Direct Coombs test & N/A & Negative & Negative & N/A \\
\hline Creatinine & N/A & $2 \mathrm{x}$ baseline & N/A & N/A \\
\hline Coagulation studies & Normal & Normal & Normal & N/A \\
\hline Terminal complement & & & & Elevated plasma concentration of sC5b-9 \\
\hline Proteinuria & $\mathrm{N} / \mathrm{A}$ & N/A & N/A & $\begin{array}{l}\text { Random urinalysis proteinuria concentration } \\
\text { of } \geq 30 \mathrm{mg} / \mathrm{dL}\end{array}$ \\
\hline Hypertension & N/A & N/A & N/A & $\begin{array}{l}\text { 3-18 years: } \mathrm{BP} \text { at } 95 \text { th percentile value for } \\
\text { age, sex and height; }>18 \text { years: BP } \\
\geq 140 / 90 \mathrm{mmHg}\end{array}$ \\
\hline
\end{tabular}

$L D H$ lactate dehydrogenase, $H b$ haemoglobin, $B P$ blood pressure 
With an increasing appreciation of how genetic predisposition underpins many diseases, focus has turned to the field of genomic medicine to help resolve disease risk and understand the biological mechanisms driving disease pathogenesis [24]. With overlapping features between aHUS and TA-TMA, genome sequencing has helped to close the gap between cited distinct entities and a reality of shared genetic aberrations in the alternative complement pathway. Jodele et al. [25], took an hypothesis-driven approach by assessing 17 genes in the alternative complement pathway, following the observation that complement activation (defined as elevated concentrations of plasma soluble C5b-9) at TA-TMA diagnosis predicts poor survival. They found that $65 \%$ of patients with TA-TMA had variants that increased alternative pathway complement activation in at least one of the 17 genes, whereas no known pathogenic variants were seen in the patients without TMA $(P<0.0001)$. Furthermore, variants in $>3$ genes were associated with higher mortality and were only seen in non-whites. This in part explains the racial disparity of TA-TMA incidence in this study and the previously described poorer outcome from HSCT in patients of African origin [26]. RNA sequencing has correlated gene variation (including variants predicted to be benign using in silico tools) with upregulation of complement activation [25]. These data indicate that dysregulated complement activation is central to the pathogenesis of TATMA and that genetic susceptibility plays a major role. Of course, this does not negate the importance of environmental stressors. For some, they may have less significant genetic susceptibility than others, yet experience stronger environmental stressors, and for others, they may be genetically vulnerable and develop TA-TMA with exposure to relatively fewer environmental stimuli. As genetic screening becomes easier, it may be possible in the future to identify those at highest risk of TMA before HSCT to allow closer follow-up and earlier therapy with complement-blocking drugs such as eculizumab $[3,27]$.

\section{External factors}

Transplant-associated thrombotic microangiopathy occurs, on average, in $5-15 \%$ of patients after allogenic HSCT and in $<1 \%$ after autologous HSCT. Pre-transplant conditioning, such as high-dose chemotherapy and total body irradiation are toxic to many cells and render the endothelium vulnerable [28]. Calcineurin inhibitors are commonly used in the immunosuppression regimen of HSCT and are directly toxic to endothelium. In a study on endothelial cells, both cyclosporine A and tacrolimus were proinflammatory; however, cyclosporine A exhibited significantly greater prothrombotic and proinflammatory effects [29]. Calcineurin inhibitors are frequently cited as a major risk factor for TA-TMA; indeed, their discontinuation has been shown to be effective in the treatment and sometimes reversal of the disease [22, 30]. A proposed mechanism has been that cyclosporine may suppress plasma levels of ADAMTS13 and limit its secretion. This theory is weakened by the $>10 \%$ ADAMTS13 activity seen in TATMA. Furthermore, there is an incongruent association between TA-TMA disease severity and serum cyclosporine levels [7, 31]. However, this does not disprove the role of calcineurin inhibitors in modifying ADAMTS13 activity and being aetiologically significant in TA-TMA, but instead does not provide significant credence that (alone) these drugs are directly causal of TA-TMA. Sirolimus, a macrocyclic lactone antibiotic, is a therapeutic and prophylactic agent against GVHD. Unlike the calcineurin inhibitors, it has not been experimentally found to damage endothelium [29]. That said, its use in combination with calcineurin inhibitors potentiates the latter's toxic effects, despite not conferring any independent risk for TA-TMA when used alone [32, 33]. Its concomitant use with cyclosporine has been shown to confer pro-necrotic and anti-angiogenic activities on endothelial cells. Some studies have shown a lower risk of GVHD and non-relapse mortality using a sirolimus/cyclosporine combination [20,34], but a large, prospective study found no difference between a combination of tacrolimus and sirolimus compared with a combination of tacrolimus and methotrexate [35].

Much like the calcineurin inhibitors, acute GVHD has been associated with a four-fold higher incidence of TA-TMA [31], but it has been difficult to distinguish GVHD as an independent risk factor for TMA due to the confounding concomitant use of calcineurin inhibitors [6]. There has been further argument that TA-TMA is a variant of GVHD; however, the strongest piece of evidence to refute this claim stems from the observation that TA-TMA occurs following autologous HSCT, and that GVHD and TA-TMA can occur independently of one another [6, 7]. TMA may be misattributed to GVHD and thus initiate an increase in immunosuppression, but increased immunosuppression neither prevents nor treats TATMA [15]. In truth, GVHD and TA-TMA are not mutually exclusive and often overlap; both are associated with endothelial injury and they share many clinical features. Although cessation of immunosuppressants such as calcineurin inhibitors can be used to treat TA-TMA, their discontinuation may worsen GVHD and/or provoke GVHD recurrence. In patients with established GVHD and TMA, it is necessary to treat both diseases concurrently $[15,36]$.

Certain viruses, notably cytomegalovirus (CMV), adenovirus, parvovirus B19, BK virus and human herpes virus 6 (HHV-6) have been associated with the development of TATMA $[37,38]$. It remains to be elucidated whether these particular viruses directly trigger TMA, or whether their presence is merely a reflection of overall disease severity post-HSCT [6]. That said, CMV and HHV-6 have been shown to directly damage endothelial cells and cause platelet aggregation [39, 40], and viral infections are a major activator of the alternative complement pathway [7]. 


\section{Pathophysiology}

There is limited consensus on the pathophysiology of TATMA, but there are a number of studies that provide sufficient data to allow the formulation of a hypothesis (Fig. 1). Most authors agree that endothelial damage is pathologically intrinsic to the disease process $[10,31$, $32,41]$. This idea was born from studies that showed that microvascular endothelial cell apoptosis is induced after exposure to TTP and diarrhoea-negative HUS plasma, associated with the rapid induction of Fas CD95 [10, 42]. In addition, scanning electron microscopy has shown endothelial cell damage in TA-TMA, and soluble VCAM-1 and E-selectin levels are significantly elevated around the onset of TMA, giving further credence to the notion that endothelial damage might be a central event in a pathological process of procoagulation and platelet aggregation that results in the clinical features of TMA [43].

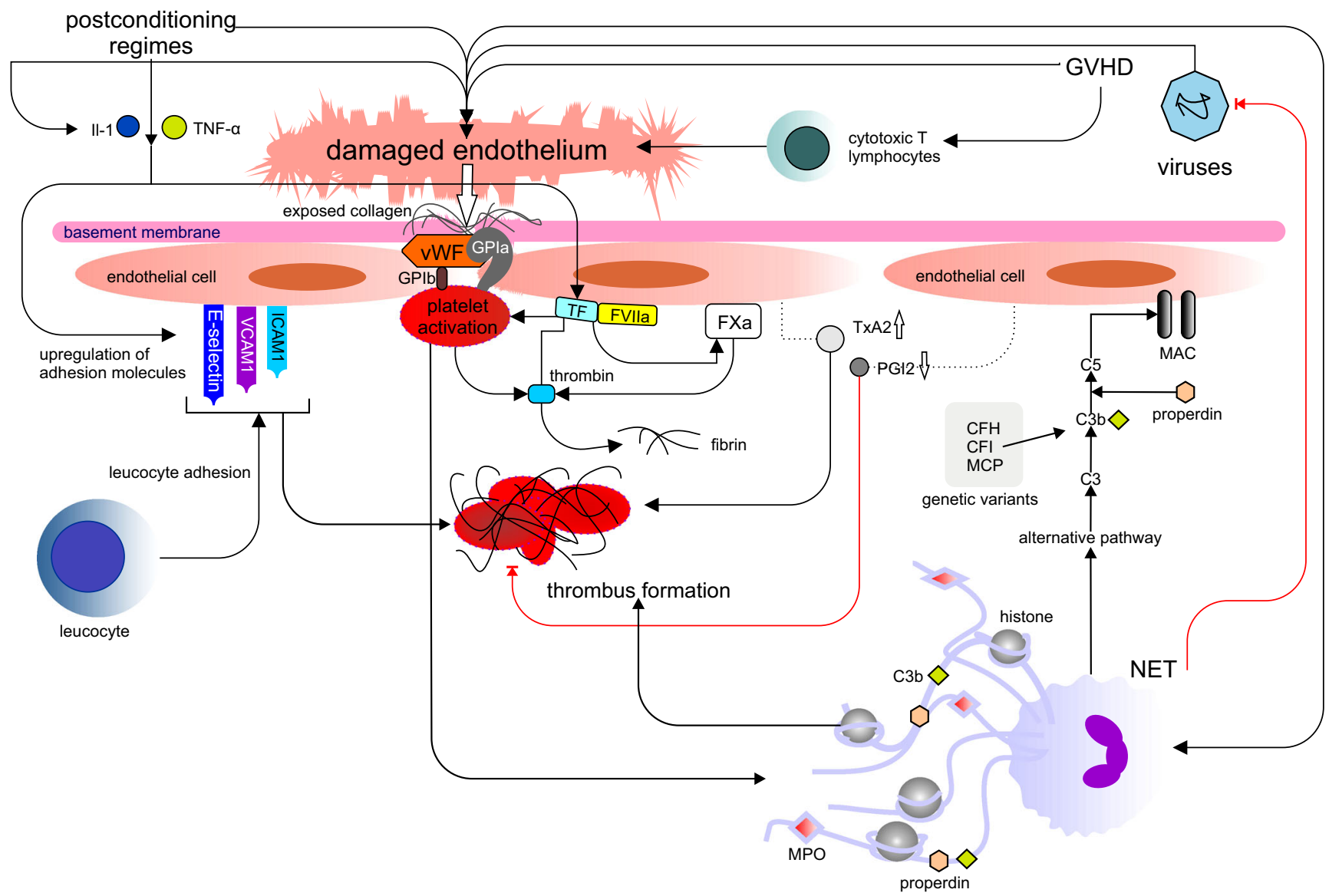

Fig. 1 Endothelial injury pathways in thrombotic microangiopathies. Extrinsic factors from haematopoietic stem cell transplantation (HSCT), such as graft versus host disease (GVHD), post-conditioning regimens and viruses can all damage endothelium, either directly, or via chemotaxis and activation of donor cytotoxic $\mathrm{T}$ lymphocytes and upregulation of proinflammatory mediators such as interleukin 1 (IL-1) and tumour necrosis factor alpha (TNF- $\alpha)$. Furthermore, activated endothelium itself promotes IL- 1 and TNF- $\alpha$, perpetuating the inflammation and damage. Endothelial injury exposes collagen and negative charges, which binds von Willebrand factor (vWF), and GPIa, which facilitates platelet activation through the platelet glycoprotein GP1b. Concomitantly, exposed tissue factor (TF) forms a complex with activated factor VIIa and activating factor $\mathrm{Xa}$, leading to the formation of thrombin. This, in turn, facilitates the further activation and aggregation of platelets, which, with the help of fibrin, forms a thrombus, capable of occluding vessels. $\mathrm{TF}$, in addition to the aforementioned cytokines TNF- $\alpha$ and IL-1, upregulate soluble adhesion molecules, such as E-selectin, vascular cellular adhesion molecule 1 (VCAM-1) and intercellular adhesion molecule 1 (ICAM-1) to promote leukocyte adhesion and further promote thrombus formation. Increased levels of thromboxane A2 (TxA2) and reduced levels of suppressed production of prostaglandin I2 (PG12) observed in TA-TMA, fail to suppress platelet aggregation precipitated by cytokine-induced endothelial activation. Neutrophil extracellular traps (NETs), capable of killing viruses and fungi, are proposed to be a fundamental component of TA-TMA, promoting endothelial damage and thrombus formation. Furthermore, complement factor $\mathrm{Bb}$ and properdin are deposited on NETs, with consequent activation of the alternative complement pathway. Factors intrinsic to the host by means of genetic variants that alter the alternative complement pathway, can result in the unrestricted formation of $\mathrm{C} 3$ convertase on the endothelial cell surface, converting $\mathrm{C} 3$ to $\mathrm{C} 3 \mathrm{a}$ and $\mathrm{C} 3 \mathrm{~b}$, and ultimately resulting in injury to the endothelium through formation of the membrane attack complex (MAC) [6]. The pathophysiology of TA-TMA is a dynamic, contemporaneous process that involves many converging pathways. Inevitably, it is likely that an admixture of extrinsic and intrinsic factors, with relative impact, controls the process and severity of TA-TMA 
Plasma markers of endothelial injury are elevated in acute GVHD, and may be explained by a direct cytotoxic donor $\mathrm{T}$ lymphocyte attack on the endothelium. Activated endothelium promotes tumour necrosis factor alpha (TNF- $\alpha$ ) and interleukin 1 (IL-1), which work synergistically to increase expression of plasminogen activator inhibitor 1 (PAI-1) and tissue factor, and TNF- $\alpha$ upregulates E-selectin, intercellular adhesion molecule 1 (ICAM-1) and platelet and endothelial adhesion molecule 1(PECAM-1 or CD31) to promote leukocyte adhesion [1]. Increased levels of IL-1, TNF- $\alpha$ and interferon gamma (IFN- $\gamma$ ) are commonly reported in the setting of acute GVHD and post-conditioning regimens [10, 32]. Suppressed production of $\mathrm{PG}_{2}$ is also observed in TA-TMA; when depleted, it fails to adequately suppress platelet aggregation precipitated by cytokine-induced endothelial activation [10, 41]. This is perhaps augmented by cyclosporine's effect on the increasing production of thromboxane $\mathrm{A} 2$ and thus inhibits the production of prostacyclin $\left(\mathrm{PGI}_{2}\right)$ [1].

The microvascular endothelium is a complex tissue that synthesises a multitude of factors governing coagulation and fibrinolysis. Such substances include tissue factor, ICAM-1, vWF, thrombomodulin, PAI-1, PG1 $1_{2}$ and nitric oxide. Variations in the abundance of these factors is shown to be associated with TMA [32, 40], either caused by intrinsic factors that render the endothelium vulnerable to activation, or by directly toxic insults as associated with HSCT. The sequence of events following endothelial damage includes intimal swelling and fibrinoid necrosis of the microvascular wall, leading to platelet aggregation, partly occluding the vessel lumen. Erythrocytes are mechanically sheared by the fibrinrich micro-thrombi, resulting in the clinical manifestations of Coombs-negative microangiopathic haemolysis, raised bilirubin, raised LDH and reduced haptoglobin.

In addition to their traditional role in the innate immunity of phagocytosis and intracellular killing of pathogens, certain neutrophils, especially low-density neutrophils, can extrude their DNA and associated peptides such as histones and myeloperoxidase to form reticular structures known as neutrophil extracellular traps (NETs) that can kill a wide variety of pathogens from viruses to fungi [44]. Although the factors leading to NETosis (the process of NET formation) are incompletely understood, we do know that NETs can damage endothelial cells [45]. Patients with a variety of TMAs have elevated levels of NETs in the plasma and remission is associated with a return to normal levels [46]. Damaged endothelium induces NET formation, which in turn drives further endothelial damage [47]. Arai et al. [16] showed an association between NET levels pre- and post-HSCT, with higher levels correlated with the development of TA-TMA and directly visualised NETs in the renal glomeruli. Furthermore, complement factor $\mathrm{Bb}$ and properdin are deposited on NETS with consequent activation of the alternative complement pathway, both on the NETs and in the serum [48].
Complement serves to provide host immunosurveillance, aiding the clearance of foreign and apoptotic cells. Under physiological conditions, circulating complement factor $\mathrm{H}$ (CFH) works together with membrane regulators by binding to host-cell surfaces and inhibiting complement attack by the alternative complement pathway [49]. In aHUS, regulatory proteins of the alternative complement pathway are frequently defective. This is either genetically determined or acquired through the presence of complement factor $\mathrm{H}$ or factor I autoantibodies, and results in unrestricted formation of $\mathrm{C} 3$ convertase on the endothelial cell surface, converting $\mathrm{C} 3$ to $\mathrm{C} 3 \mathrm{a}$ and $\mathrm{C} 3 \mathrm{~b}$. In turn, this promotes the formation of $\mathrm{C} 5$ convertase, resulting in the formation of the membrane attack complex and the anaphylatoxin $\mathrm{C} 5 \mathrm{a}$, which cause injury to the endothelium [6]. Interestingly, the complement staining pattern of TA-TMA resembles that of aHUS, with $\mathrm{C} 4 \mathrm{~d}$ deposition localised to the glomeruli and arterioles [17], offering an historical record of complement activation, although it should be noted that $\mathrm{C} 4 \mathrm{~d}$ staining is also seen in a small number of patients without evidence of TA-TMA [8]. This suggests that TA-TMA might represent aHUS in the setting of HSCT and this theory is certainly supported by the enrichment of variants in complement genes seen in TA-TMA versus HSCT without TMA [25]. These findings suggest that a genetically weakened complement regulatory system is vulnerable to HSCTrelated factors that can further activate the complement system and directly damage microvascular endothelium. The role of complement is further supported by the observation that elevated plasma concentrations of the terminal complement complex, C5b-9, correlate with severe disease and poor outcome, in addition to reports of the beneficial effect of eculizumab treatment in patients with TA-TMA [8].

It can therefore be hypothesised that in certain patients, factors such as genetic predisposition, underlying disease, conditioning regimen, GVHD or infection might lead to exuberant NETosis and/or reduced clearance of NETs. This leads to endothelial damage and complement activation. In patients with genetic variants reducing inhibition of alternative pathway complement activation, this can lead to life-threatening TMA.

\section{How TA-TMA specifically affects the kidney}

Renal biopsy specimens of TA-TMA patients show mesangiolysis and loss of endothelial cells, with subendothelial expansion and luminal occlusion by fragmented erythrocytes, fibrin deposition and necrotic products [31]. C4d deposition in the glomerular and peritubular capillaries is common to TA-TMA and aHUS [17]. Inflammatory infiltrates, including natural killer cells, CD3+ and CD8+ T cells and cytotoxic T cells, have been found within the kidney glomeruli, interstitium and tubules [31]. The resulting clinical presentation includes proteinuria, 
hypertension and decreased glomerular filtration rate (GFR), yet these may also be observed in transplant recipients without TA-TMA, i.e. those exposed to nephrotoxic and hypertensioninducing medicines and certain bacterial and viral infections [6]. That said, renal TA-TMA should be suspected when HSCT recipients require more antihypertensive therapy than would be expected for GVHD prophylaxis or treatment, and should especially be considered in patients requiring more than two antihypertensive therapies [6] or with nephroticrange proteinuria [11].

\section{Extra-renal features}

Transplant-associated thrombotic microangiopathy is not merely a renal disease; vascular involvement of extra-renal organs such as the heart, lungs and gastrointestinal tract have all been reported in TA-TMA and multi-organ involvement confers a higher morbidity and mortality $[15,18]$.

\section{Heart and lungs}

The incidence of cardiac and pulmonary complications postHSCT has been reported in as many as $30 \%$ of cases, and includes pericardial effusion (PEF), elevated right ventricular (RV) pressure and reduced left ventricular function. Raised right-sided pressures have been significantly associated with TA-TMA; indeed, in a single-centre prospective study, Dandoy et al. found that nearly $70 \%$ of patients with elevated $\mathrm{RV}$ pressures, as detected by echocardiogram on day 7 postHSCT, were subsequently diagnosed with TA-TMA [50]. Furthermore, all patients with both PEF and raised RV pressures had concomitant TA-TMA, potentially representing a polyserositis [18]. Histological evidence for pulmonary arteriolar microangiopathy includes injured endothelium, microthrombosis and schistocyte extravasation into the pulmonary interstitium [6]. As a result, pulmonary artery pressures rise and RV failure may ensue. These authors propose that elevated RV pressures may indicate early pulmonary vascular injury that can precede haematologically detectable TATMA by current diagnostic criteria. The importance of identifying pulmonary involvement early is imperative, as posttransplant survival at 1 year is statistically worse in those with cardiac and pulmonary involvement [50]. Left untreated, pulmonary hypertension in the presence of TA-TMA has a high mortality rate [6].

\section{Gastrointestinal tract}

Severe diarrhoea, abdominal pain and bleeding are common and serious adverse events post-HSCT, frequently blamed on infection or gut GVHD. However, in many cases, these symptoms, most notably abdominal pain and bleeding, are refractory to increased immunosuppression, implicating the gastrointestinal (GI) tract as another target for TMA [51]. As GVHD and TA-TMA can occur concurrently, increased immunosuppression tends to treat the voluminous diarrhoea caused by GVHD, whilst leaving the bowel susceptible to ischaemia by TMA. In fact, biopsies from colonoscopy studies of clinically diagnosed acute gut GVHD have been reported to show histological evidence of TA-TMA in $>90 \%$ of patients, with as few as $30 \%$ of patients actually having histological evidence of GVHD [52]. Therefore, abdominal pain and rectal bleeding should raise the possibility of TA-TMA. Histologically, gut TMA presents as endothelial cell separation from the vascular wall, aggregation of intraluminal fibrin, microthrombi and schistocytes, with denudation of mucosa and loss of glands [51]. Differentiating gut GVHD from gut TMA is difficult, not least because they share some histological features and can occur concomitantly $[6,51]$. The histological unpicking of GI disease aetiology is essential, as treatment regimens are antipodal; calcineurin inhibitors treat GVHD, whilst their discontinuation is one of the mainstay treatments for TA-TMA [6].

\section{Central nervous system}

Central nervous system (CNS) involvement in TA-TMA is not fully understood. Patients with TA-TMA often have neurological symptoms, including headache, seizures, visual disturbances, confusion and hallucinations, but many of these symptoms may be attributed to metabolic disturbances or hypertension complicating TA-TMA [6]. If hypertension is uncontrolled, there is a risk of posterior reversible encephalopathy syndrome (PRES), an acute encephalopathy syndrome characterised by seizures, altered mental status and visual disturbances [53]. PRES, like TMA, is a disease of endothelial injury. It occurs because of abrupt changes in blood pressure or from direct cytotoxicity of the blood-brain barrier. Radiological findings include bilateral vasogenic oedema concentrated in the subcortical parieto-occipital regions. As the name states, PRES is considered reversible; however, in some cases cerebral haemorrhage ensues, which can lead to significant morbidity or mortality from brain infarction and brainstem compression respectively [54].

\section{Diagnosis}

Currently, there are no universally accepted diagnostic criteria for TA-TMA; nevertheless, attempts have been made to create a framework for diagnosis, and broadly speaking, thrombocytopaenia, schistocytosis, increased LDH, decreased haptoglobin, a negative direct Coombs test with normal coagulation studies, and exclusion of TTP by $>10 \%$ ADAMTS13 levels are accepted clinical features that warrant diagnosis 
(Table 2). The difficulty in creating diagnostic criteria is that TA-TMA shares many clinical features with sequelae of HSCT, limiting the specificity of any one feature. Taking each clinical criterion in turn, it can be argued that alternative pathological processes are responsible. For example, thrombocytopaenia may occur as a result of delayed megakaryocyte engraftment, infection, GVHD and myelosuppressive therapies [1]. Schistocytosis is not uncommon in transplant patients, and may be attributed to infection, underlying haematological malignancies or disseminated intravascular coagulation [7]. Furthermore, the assessment for schistocytes is a somewhat subjective test requiring haematological screening of peripheral blood smears, and reporting may vary among and within laboratories [55]. Haptoglobin and LDH levels are again non-specific findings and institutional laboratory values vary.

To complicate matters further, the presentation of TA-TMA is extremely variable. Disease severity can range from asymptomatic low-grade disease confined to the kidneys, to fulminant, systemic disease involving the lungs, heart, gut and brain $[1,6]$. That said, there should be a high index of suspicion for TA-TMA with multi-organ involvement post-HSCT. Of course, detecting TA-TMA before there is multi-organ failure is of paramount importance, and arguably the current diagnostic criteria are more sensitive to established disease, i.e. schistocytes on peripheral blood smear may represent a late sign of vascular injury [50], and decreased haptoglobin levels and doubling of serum creatinine present 2 and 4 weeks respectively after diagnosis [15]. Consequently, research has

Table 2 List of genes related to transplant-associated thrombotic microangiopathy (TA-TMA)

TA-TMA-related genes

$\mathrm{CFH}$

CFHRI

CFHR3

CFHR4

CFHR5

CD55

CD59

CD46

CFI

$C F B$

CFP

C5

ADAMTS13

$C F D$

$C 3$

$C 4 B P A$

$T H B D$ focused on finding early markers that may indicate TATMA. Jodele et al. [6] suggest that elevated LDH, proteinuria and hypertension might be early markers of disease, and might precede diagnosis by 10-14 days [15]. Statistically significantly more patients with TA-TMA versus without developed high random urine protein:creatinine ratios and required more anti-hypertensive therapy [55]. Elevated sC5b-9 levels provide another early marker of TA-TMA that often precedes haematological markers; however, it is not a readily available clinical test. Other suggested early diagnostic markers include elevated RV pressures and post-transplant pericardial effusion. Dandoy et al. suggest that echocardiographic detection of pulmonary hypertension might be a good and sensitive screening tool [50].

Once TA-TMA is established, patients can be tested for complement factor $\mathrm{H}$ autoantibodies and mutations in genes associated with the complement pathway, but diagnosis should not depend on the demonstration of deleterious genetic variants. When testing DNA, it is important to ensure that the DNA is truly representative of the recipient. After stem cell transplantation and engraftment, DNA extracted from the recipient's blood reflects the donor DNA; therefore, DNA should be obtained either from a stored sample before transplant or from a tissue site other than blood. Histology can support the diagnosis, but is not possible for every individual, particularly in the setting of thrombocytopaenia.

\section{Treatment}

Therapeutic approaches to TA-TMA include supportive therapy and targeted disease therapy. First-line therapy should include the minimisation of factors that may precipitate TATMA. Examples include withdrawal of calcineurin inhibitors and/or co-therapy with sirolimus, although this should be measured against the risk of precipitating GVHD, which is itself an independent risk factor for TA-TMA. Adequate antibiotic therapy is essential to minimise potentially fatal infections in immunocompromised patients, as is aggressive antihypertensive therapy and renal replacement therapy $[6,21]$.

\section{Plasma exchange}

The therapeutic use of plasma exchange in TA-TMA lacks convincing evidence. Although effective in TTP, its use in TA-TMA yields limited benefit, with response rates below $50 \%$ [21]. In TTP, plasma exchange is effective because it removes autoantibodies that deplete ADAMTS13 and replenishes supplies. In TA-TMA, and indeed aHUS, the response to plasma exchange is limited, although its efficacy is possibly influenced by confounding factors such as the time of disease presentation post-HSCT [56], GVHD [57] and timing of clinical intervention [58]. Moreover, clinicians must be cautious 
of pseudo-responses, reflected by improvements in haematological parameters such as platelet count and haptoglobin levels; fresh frozen plasma is rich in complement regulatory proteins that can transiently appear as if the disease process is relenting, when, in fact, despite response rates between 37 and $55 \%$, the pathological process continues and mortality rates remain high at $80 \%[1,14,58,59]$. Moreover, plasma exchange harbours adverse effects, such as alloimmunisation, thrombosis, pneumothorax, pericardial tamponade, serum sickness, vascular catheter infections, and infusion reactions, such as anaphylaxis and transfusion-related acute lung injury [7].

\section{Immunotherapies and anti-thrombotic therapies}

The anti-CD20 monoclonal antibody therapy, rituximab, has proven efficacy in TTP with ADAMTS13 activity levels $<10 \%$ [7] and in the prevention of aHUS with CFH autoantibodies after kidney transplantation [15]. The empirical therapeutic use of rituximab in TA-TMA has been largely positive, achieving remission in patients without suppressed ADAMTS13 activity, most likely by acting as an immunomodulatory agent [60]. Of course, the possibility of reporting bias cannot be excluded, as cases with poor outcomes may be reported less commonly. Its mechanism of action in TA-TMA is not fully understood, although it is hypothesised that it may act by depleting CD20+ B cell precursors, thus attenuating Tcell activation and cytokine release [60]. Defibrotide is a polydeoxyribonucleotide that has shown promise as a potential TA-TMA therapy through its action on platelet aggregation and endothelial protection against calcineurin inhibitors, without a significant effect on systemic coagulation $[29,55$, 61]. Corti showed defibrotide to be beneficial in approximately $50 \%$ of patients post-HSCT [61]. That said, the studies on defibrotide lack substantial numbers and data to ratify some of the claims made on its efficacy.

\section{Complement targeted therapies}

Perhaps the most promising therapy for TA-TMA is eculizumab, a terminal complement inhibitor that is highly effective in treating aHUS. With increasing evidence for the role of dysregulated activation of the alternative complement pathway underpinning endothelial damage that drives TMA, focus has turned to drugs that act to inhibit the complement system.

Eculizumab is a recombinant, fully humanised, monoclonal antibody that binds complement $\mathrm{C} 5$ and blocks production of proinflammatory $\mathrm{C} 5 \mathrm{a}$ and the membrane attack complex $\mathrm{C} 5 \mathrm{~b}-9$ [27]. Its use in aHUS has proved life-saving for many patients. With considerable overlap between aHUS and TA-TMA both genetically and histologically, eculizumab has been used for both the prevention and treatment of TA-TMA. However, its use and efficacy are not interchangeable and the drug exhibits varying pharmacokinetics and pharmacodynamics. Thus far, the outcomes have been favourable. Indeed, Jodele et al. showed improved survival in a small cohort of patients with high-risk TA-TMA treated with eculizumab compared with alternative treatments. One-year overall survival was $62 \%$ vs $9 \%$ $(P=0.0007)$, although in some patients it has not been possible to achieve therapeutic plasma concentrations. For the best results, eculizumab administration in TA-TMA requires a personalised drug-dosage regimen that is titrated against a variable drug clearance, which is determined by disease severity [62]. Importantly, available evidence suggests that eculizumab therapy might not need to be continued long term and can safely be stopped after TA-TMA resolution, offering an effective and curative treatment. It is also important to give TA-TMA patients on eculizumab prophylactic antibiotics to prevent meningococcal disease. Usually, a polyvalent meningococcal vaccine is administered 2 weeks before the first dose of eculizumab, as complement blockade renders the immune system vulnerable to meningococci. As post-transplant recipients are severely immunocompromised, they are unable to mount a vaccine response until their adaptive immune system is reconstituted. Reassuringly, evidence has shown that with adequate antibiotic prophylaxis, there is no increased risk of meningococcal disease in non-vaccinated eculizumab recipients [63].

\section{Outcome/prognosis}

Transplant-associated thrombotic microangiopathy causes significant morbidity and mortality, with reported death rates as high as $75 \%$ within 3 months of diagnosis [12]. Research has focused on finding early prognostic factors to aid faster diagnosis, quicker intervention and predict those at highest risk of an unfavourable disease trajectory.

Factors associated with poor outcomes include proteinuria ( $>30 \mathrm{mg} / \mathrm{dL}$ ) and raised plasma levels of sC5b-9 at diagnosis [11]. Jodele et al. showed that patients with elevated plasma concentrations of the terminal complement complex sC5b-9 and proteinuria at diagnosis had an $84 \%$ non-relapse mortality rate 1 year post-HSCT; all patients without proteinuria and elevated sC5b-9 survived [6]. Pulmonary hypertension is another poor prognostic factor and mortality rates reach $80 \%$ [55]. However, with evidence that pulmonary hypertension may precede haematological diagnostic parameters, these rates may decrease if prompt treatment is administered.

\section{Conclusion}

Transplant-associated thrombotic microangiopathy is a multifaceted disease process underpinned by host factors and external stressors. Now understood as a separate entity to TTP, but as a tissue microangiopathy that closely mimics aHUS, we 
are beginning to gain a better understanding of the pathogenesis of this devastating disease. The discovery of NETs and their role in endothelial damage, thrombus formation and complement activation may prove to be an integral component of TMA pathophysiology. Despite efforts to improve outcomes over recent years with biological drugs such as eculizumab, TA-TMA remains a potentially fatal complication of HSCT. Identifying those at greatest risk of TA-TMA and recognising the early warning signs of disease will inevitably improve survival and outcomes.

\section{Key summary points}

1. Transplant-associated thrombotic microangiopathy is a frequent complication of stem cell transplants, sharing clinical features with GVHD. It is important to distinguish between GVHD and TA-TMA and where there is an overlap, it is essential to treat both diseases.

2. Dysregulated complement activation plays a central role in the pathogenesis of TA-TMA. Multiple factors, both genetic and environmental, may contribute to abnormal complement activation.

3. Transplant-associated thrombotic microangiopathy strongly overlaps with aHUS, sharing many genetic disease risk alleles. Eculizumab therapy has proved successful in the treatment of both aHUS and TA-TMA, and in many cases provides a definitive cure. For TA-TMA specifically, patient-specific drug dose regimens are required to ensure optimal pharmacokinetics in a disease that exhibits varying drug clearance in changing states of disease activity.

4. Endothelial damage is intrinsic to the pathogenesis of TATMA. There is emerging evidence that neutrophil extracellular traps (NETs) may play a crucial role in the pathophysiology of endothelial damage and complement activation.

\section{Questions (answers are provided following the reference list)}

\section{Which of the following statements is false?}

a) Transplant-associated thrombotic microangiopathy is more common following autologous haematopoietic stem cell transplantation.

b) Calcineurin inhibitor treatment may predispose patients to TA-TMA.

c) Some patients may be genetically predisposed to developing TA-TMA.

d) Conditioning regimens may predispose to TMA. e) Pulmonary hypertension 1 week after transplantation may indicate a high risk of later development of TA-TMA.

2. Which two of the following statements are false?

a) Low platelet count does not confirm a diagnosis of TATMA.

b) Diarrhoea in a recipient of a haematopoietic stem cell transplant can be caused by infection or graft versus host disease, but not by TA-TMA.

c) Patients with TA-TMA may have multisystem disease, including cardiac and CNS involvement.

d) Diagnosis of TA-TMA can be aided by biopsy of affected tissues.

e) There are no internationally accepted criteria for establishing a diagnosis of TA-TMA.

3. Which of the following is NOT an appropriate therapeutic choice in a patient with TA-TMA:

a) Treatment with eculizumab

b) Treatment with rituximab

c) Treatment with defibrotide

d) Treatment with cyclosporine and sirolimus

4. Which two of the following statements are true?

a) Untreated, TA-TMA can be a devastating disease with a very high mortality rate.

b) Plasma exchange is useful, as it replenishes ADAMTS13 levels.

c) If eculizumab treatment is started, it needs to be continued throughout life.

d) Patients with TA-TMA almost all have very low plasma concentrations of complement $\mathrm{C} 3$.

e) The presence of hypertension and heavy proteinuria together strongly suggests a diagnosis of TA-TMA.

5. Which of the following statements is false?

a) Endothelial damage caused by conditioning regimens may contribute to the pathogenesis of TA-TMA.

b) Complement activation, especially the alternative pathway, appears to play an important role in the pathogenesis of TA-TMA.

c) NETosis is an attempt by the body to combat TA-TMA and therapies that promote it are likely to be effective at treating TA-TMA.

d) Platelet activation probably plays an important role in the pathogenesis of TA-TMA.

e) Neutrophil extracellular traps can activate complement and damage endothelial cells. 


\section{Compliance with ethical standards}

Conflicts of interest RDG has received several honoraria from Alexion for lectures on atypical HUS and the use of eculizumab.

Open Access This article is distributed under the terms of the Creative Commons Attribution 4.0 International License (http:// creativecommons.org/licenses/by/4.0/), which permits unrestricted use, distribution, and reproduction in any medium, provided you give appropriate credit to the original author(s) and the source, provide a link to the Creative Commons license, and indicate if changes were made.

\section{References}

1. Daly A, Xenocostas A, Lipton J (2002) Transplantation-associated thrombotic microangiopathy: twenty-two years later. Bone Marrow Transplant 30:709-715

2. Jodele S, Licht C, Goebel J, Dixon BP, Zhang K, Sivakumaran TA, Davies SM, Pluthero FG, Lu L, Laskin BL (2013) Abnormalities in the alternative pathway of complement in children with hematopoietic stem cell transplant-associated thrombotic microangiopathy. Blood 122:2003-2007

3. De Fontbrune FS, Galambrun C, Sirvent A, Huynh A, Faguer S, Nguyen S, Bay J-O, Neven B, Moussi J, Simon L (2015) Use of eculizumab in patients with allogeneic stem cell transplantassociated thrombotic microangiopathy: a study from the SFGMTC. Transplantation 99:1953-1959

4. Holler E, Kolb H, Hiller E, Mraz W, Lehmacher W, Gleixner B, Seeber C, Jehn U, Gerhartz H, Brehm G (1989) Microangiopathy in patients on cyclosporine prophylaxis who developed acute graftversus-host disease after HLA-identical bone marrow transplantation. Blood 73:2018-2024

5. Atkinson K, Biggs J, Hayes J, Ralston M, Dodds A, Concannon A, Naidoo D (1983) Cyclosporin A associated nephrotoxicity in the first 100 days after allogeneic bone marrow transplantation: three distinct syndromes. Br J Haematol 54:59-67

6. Jodele S, Laskin BL, Dandoy CE, Myers KC, El-Bietar J, Davies SM, Goebel J, Dixon BP (2015) A new paradigm: diagnosis and management of HSCT-associated thrombotic microangiopathy as multi-system endothelial injury. Blood Rev 29:191-204

7. Chapin J, Shore T, Forsberg P, Desman G, Van Besien K, Laurence J (2014) Hematopoietic transplant-associated thrombotic microangiopathy: case report and review of diagnosis and treatments. Clin Adv Hematol Oncol 12:565-573

8. Jodele S, Fukuda T, Vinks A, Mizuno K, Laskin BL, Goebel J, Dixon BP, Teusink A, Pluthero FG, Lu L (2014) Eculizumab therapy in children with severe hematopoietic stem cell transplantationassociated thrombotic microangiopathy. Biol Blood Marrow Transplant 20:518-525

9. Tsai H-M, Lian EC-Y (1998) Antibodies to von Willebrand factorcleaving protease in acute thrombotic thrombocytopenic purpura. $\mathrm{N}$ Engl J Med 339:1585-1594

10. Batts E, Lazarus H (2007) Diagnosis and treatment of transplantation-associated thrombotic microangiopathy: real progress or are we still waiting? Bone Marrow Transplant 40:709-719

11. Jodele S, Davies SM, Lane A, Khoury J, Dandoy C, Goebel J, Myers K, Grimley M, Bleesing J, El-Bietar J (2014) Diagnostic and risk criteria for HSCT-associated thrombotic microangiopathy: a study in children and young adults. Blood 124:645-653

12. Ho VT, Cutler C, Carter S, Martin P, Adams R, Horowitz M, Ferrara J, Soiffer R, Giralt S (2005) Blood and marrow transplant clinical trials network toxicity committee consensus summary: thrombotic microangiopathy after hematopoietic stem cell transplantation. Biol Blood Marrow Transplant 11:571-575

13. Pettitt A, Clark R (1994) Thrombotic microangiopathy following bone marrow transplantation. Bone Marrow Transplant 14:495504

14. Fuge R, Bird JM, Fraser A, Hart D, Hunt L, Cornish JM, Goulden N, Oakhill A, Pamphilon DH, Steward CG (2001) The clinical features, risk factors and outcome of thrombotic thrombocytopenic purpura occurring after bone marrow transplantation. Br J Haematol 113:58-64

15. Laskin BL, Goebel J, Davies SM, Jodele S (2011) Small vessels, big trouble in the kidneys and beyond: hematopoietic stem cell transplantation-associated thrombotic microangiopathy. Blood 118:1452-1462

16. Arai Y, Yamashita K, Mizugishi K, Watanabe T, Sakamoto S, Kitano T, Kondo T, Kawabata H, Kadowaki N, Takaori-Kondo A (2013) Serum neutrophil extracellular trap levels predict thrombotic microangiopathy after allogeneic stem cell transplantation. Biol Blood Marrow Transplant 19:1683-1689

17. Laskin BL, Maisel J, Goebel J, Yin HJ, Luo G, Khoury JC, Davies SM, Jodele S (2013) Renal arteriolar C4d deposition: a novel characteristic of hematopoietic stem cell transplantation-associated thrombotic microangiopathy. Transplantation 96:217-223

18. Lerner D, Dandoy C, Hirsch R, Laskin B, Davies S, Jodele S (2014) Pericardial effusion in pediatric SCT recipients with thrombotic microangiopathy. Bone Marrow Transplant 49:862-863

19. Chappell M, Keeling D, Prentice H, Sweny P (1988) Haemolytic uraemic syndrome after bone marrow transplantation: an adverse effect of total body irradiation? Bone Marrow Transplant 3:339-347

20. Fortin MC, Raymond MA, Madore F, Fugère JA, Pâquet M, StLouis G, Hébert MJ (2004) Increased risk of thrombotic microangiopathy in patients receiving a cyclosporin-sirolimus combination. Am J Transplant 4:946-952

21. Stavrou E, Lazarus HM (2010) Thrombotic microangiopathy in haematopoietic cell transplantation: an update. Mediterr J Hematol Infect Dis 2:e2010033

22. Zeigler Z, Shadduck R, Nemunaitis J, Andrews D, Rosenfeld C (1995) Bone marrow transplant-associated thrombotic microangiopathy: a case series. Bone Marrow Transplant 15:247-253

23. Martinez MT, Bucher C, Stussi G, Heim D, Buser A, Tsakiris D, Tichelli A, Gratwohl A, Passweg J (2005) Transplant-associated microangiopathy (TAM) in recipients of allogeneic hematopoietic stem cell transplants. Bone Marrow Transplant 36:993-1000

24. Seaby EG, Pengelly RJ, Ennis S (2015) Exome sequencing explained: a practical guide to its clinical application. Brief Funct Genomics 15:374-384

25. Jodele S, Zhang K, Zou F, Laskin B, Dandoy CE, Myers KC, Lane A, Meller J, Medvedovic M, Chen J (2016) The genetic fingerprint of susceptibility for transplant-associated thrombotic microangiopathy. Blood 127:989-996

26. Baker KS, Davies SM, Majhail NS, Hassebroek A, Klein JP, Ballen KK, Bigelow CL, Frangoul HA, Hardy CL, Bredeson C (2009) Race and socioeconomic status influence outcomes of unrelated donor hematopoietic cell transplantation. Biol Blood Marrow Transplant 15:1543-1554

27. Legendre CM, Licht C, Muus P, Greenbaum L, Babu S, Bedrosian C, Bingham C, Cohen D, Delmas Y, Douglas K (2013) Terminal complement inhibitor eculizumab in atypical hemolytic-uremic syndrome. N Engl J Med 368:2169-2181

28. Shimoni A, Yeshurun M, Hardan I, Avigdor A, Ben-Bassat I, Nagler A (2004) Thrombotic microangiopathy after allogeneic stem cell transplantation in the era of reduced-intensity conditioning: the incidence is not reduced. Biol Blood Marrow Transplant 10:484-493 
29. Carmona A, Díaz-Ricart M, Palomo M, Molina P, Pino M, Rovira M, Escolar G, Carreras E (2013) Distinct deleterious effects of cyclosporine and tacrolimus and combined tacrolimus-sirolimus on endothelial cells: protective effect of defibrotide. Biol Blood Marrow Transplant 19:1439-1445

30. Sarkodee-Adoo C, Sotirescu D, Sensenbrenner L, Rapoport AP, Cottler-Fox M, Tricot G, Ruehle K, Meisenberg B (2003) Thrombotic microangiopathy in blood and marrow transplant patients receiving tacrolimus or cyclosporine A. Transfusion (Paris) 43:78-84

31. Changsirikulchai S, Myerson D, Guthrie KA, McDonald GB, Alpers CE, Hingorani SR (2009) Renal thrombotic microangiopathy after hematopoietic cell transplant: role of GVHD in pathogenesis. Clin J Am Soc Nephrol 4:345-353

32. Cooke KR, Jannin A, Ho V (2008) The contribution of endothelial activation and injury to end-organ toxicity following allogeneic hematopoietic stem cell transplantation. Biol Blood Marrow Transplant 14:23-32

33. Robson M, Côte I, Abbs I, Koffman G, Goldsmith D (2003) Thrombotic micro-angiopathy with sirolimus-based immunosuppression: potentiation of calcineurin-inhibitor-induced endothelial damage? Am J Transplant 3:324-327

34. Perez-Simón JA, Martino R, Parody R, Cabrero M, Lopez-Corral L, Valcarcel D, Martinez C, Solano C, Vazquez L, MárquezMalaver FJ (2013) The combination of sirolimus plus tacrolimus improves outcome after reduced-intensity conditioning, unrelated donor hematopoietic stem cell transplantation compared with cyclosporine plus mycofenolate. Haematologica 98:526-532

35. Cutler C, Logan B, Nakamura R, Johnston L, Choi S, Porter D, Hogan WJ, Pasquini M, MacMillan ML, Hsu JW (2014) Tacrolimus/sirolimus vs tacrolimus/methotrexate as GVHD prophylaxis after matched, related donor allogeneic HCT. Blood 124: 1372-1377

36. Rotz SJ, Dandoy CE, Davies SM (2017) ST2 and endothelial injury as a link between GVHD and microangiopathy. N Engl J Med 376: 1189-1190

37. Belford A, Myles O, Magill A, Wang J, Myhand RC, Waselenko JK (2004) Thrombotic microangiopathy (TMA) and stroke due to human herpesvirus-6 (HHV-6) reactivation in an adult receiving highdose melphalan with autologous peripheral stem cell transplantation. Am J Hematol 76:156-162

38. Murer L, Zacchello G, Bianchi D, Dall'Amico R, Montini G, Andreetta B, Perini M, DOSSI EC, Zanon G, Zacchello F (2000) Thrombotic microangiopathy associated with parvovirus B 19 infection after renal transplantation. J Am Soc Nephrol 11:1132-1137

39. Rahbar A, Söderberg-Nauclér C (2005) Human cytomegalovirus infection of endothelial cells triggers platelet adhesion and aggregation. J Virol 79:2211-2220

40. Takahashi H, Hanano M, Wada K, Tatewaki W, Niwano H, Shibata A, Tsubouchi J, Nakano M, Nakamura T (1991) Circulating thrombomodulin in thrombotic thrombocytopenic purpura. Am J Hematol 38:174-177

41. Cohen H, Bull H, Seddon A, Enayat M, Hill F, Woolf N, Machin S (1989) Vascular endothelial cell function and ultrastructure in thrombotic microangiopathy following allogeneic bone marrow transplantation. Eur J Haematol 43:207-214

42. Mitra D, Jaffe EA, Weksler B, Hajjar KA, Soderland C, Laurence J (1997) Thrombotic thrombocytopenic purpura and sporadic hemolytic-uremic syndrome plasmas induce apoptosis in restricted lineages of human microvascular endothelial cells. Blood 89:12241234

43. Matsuda Y, Hara J, Osugi Y, Tokimasa S, Fujisaki H, Takai K, Ohta H, Kawa-Ha K, Okada S (2001) Serum levels of soluble adhesion molecules in stem cell transplantation-related complications. Bone Marrow Transplant 27:977-982
44. Hasler P, Giaglis S, Hahn S (2016) Neutrophil extracellular traps in health and disease. Swiss Med Wkly 146:w14352

45. Saffarzadeh M, Juenemann C, Queisser MA, Lochnit G, Barreto G, Galuska SP, Lohmeyer J, Preissner KT (2012) Neutrophil extracellular traps directly induce epithelial and endothelial cell death: a predominant role of histones. PLoS One 7:e32366

46. Fuchs TA, Hovinga JAK, Schatzberg D, Wagner DD, Lämmle B (2012) Circulating DNA and myeloperoxidase indicate disease activity in patients with thrombotic microangiopathies. Blood 120:1157-1164

47. Gupta AK, Joshi MB, Philippova M, Erne P, Hasler P, Hahn S, Resink TJ (2010) Activated endothelial cells induce neutrophil extracellular traps and are susceptible to NETosis-mediated cell death. FEBS Lett 584:3193-3197

48. Wang H, Wang C, Zhao MH, Chen M (2015) Neutrophil extracellular traps can activate alternative complement pathways. Clin Exp Immunol 181:518-527

49. Ricklin D, Cines DB (2013) TMA: beware of complements. Blood 122:1997-1999

50. Dandoy CE, Davies SM, Hirsch R, Chima RS, Paff Z, Cash M, Ryan TD, Lane A, El-Bietar J, Myers KC (2015) Abnormal echocardiography 7 days after stem cell transplantation may be an early indicator of thrombotic microangiopathy. Biol Blood Marrow Transplant 21:113-118

51. El-Bietar J, Warren M, Dandoy C, Myers KC, Lane A, Wallace G, Davies SM, Jodele S (2015) Histologic features of intestinal thrombotic microangiopathy in pediatric and young adult patients after hematopoietic stem cell transplantation. Biol Blood Marrow Transplant 21:1994-2001

52. Inamoto Y, Ito M, Suzuki R, Nishida T, Iida H, Kohno A, Sawa M, Murata M, Nishiwaki S, Oba T (2009) Clinicopathological manifestations and treatment of intestinal transplant-associated microangiopathy. Bone Marrow Transplant 44:43-49

53. Staykov D, Schwab S (2011) Posterior reversible encephalopathy syndrome. J Intensive Care Med 27:11-24

54. Fugate JE, Rabinstein AA (2015) Posterior reversible encephalopathy syndrome: clinical and radiological manifestations, pathophysiology, and outstanding questions. Lancet Neurol 14:914-925

55. Uderzo C, Jodele S, El Missiry M, Ciceri F, Bacigalupo A, Corbacioglu S (2014) Transplant-associated thrombotic microangiopathy (TA-TMA) and consensus based diagnostic and therapeutic recommendations: which TA-TMA patients to treat and when? J Bone Marrow Res 2:152

56. Mulay S, Kreuter JD, Bryant SC, Elliott MA, Hogan WJ, Winters JL, Gastineau DA (2015) Outcomes of plasma exchange in patients with transplant-associated thrombotic microangiopathy based on time of presentation since transplant. J Clin Apher 30:147-153

57. Kennedy G, Kearey N, Bleakley S, Butler J, Mudie K, Durrant S (2010) Transplantation-associated thrombotic microangiopathy: effect of concomitant GVHD on efficacy of therapeutic plasma exchange. Bone Marrow Transplant 45:699-704

58. Jodele S, Laskin BL, Goebel J, Khoury JC, Pinkard SL, Carey PM, Davies SM (2013) Does early initiation of therapeutic plasma exchange improve outcome in pediatric stem cell transplantassociated thrombotic microangiopathy? Transfusion (Paris) 53: 661-667

59. Riedl M, Fakhouri F, Le Quintrec M, Noone DG, Jungraithmayr TC, Fremeaux-Bacchi V, Licht C (2014) Spectrum of complementmediated thrombotic microangiopathies: pathogenetic insights identifying novel treatment approaches. Semin Thromb Hemost 40:444-464

60. Ostronoff M, Ostronoff F, Calixto R, Florencio R, Florencio M, Domingues M, Maior AS, Sucupira A, Tagliari C (2007) Lifethreatening hemolytic-uremic syndrome treated with rituximab in 
an allogeneic bone marrow transplant recipient. Bone Marrow Transplant 39:649-651

61. Corti P (2002) Defibrotide as a promising treatment for thrombotic thrombocytopenic purpura in patients undergoing bone marrow transplantation. Bone Marrow Transplant 29:542-543

62. Jodele S, Fukuda T, Mizuno K, Vinks AA, Laskin BL, Goebel J, Dixon BP, Chima RS, Hirsch R, Teusink A, Lazear D, Lane A, Myers KC, Dandoy CE, Davies SM (2016) Variable eculizumab clearance requires pharmacodynamic monitoring to optimize therapy for thrombotic microangiopathy after hematopoietic stem cell transplantation. Biol Blood Marrow Transplant 22:307-315
63. Jodele S, Dandoy CE, Danziger-Isakov L, Myers KC, El-Bietar J, Nelson A, Wallace G, Teusink-Cross A, Davies SM (2016) Terminal complement blockade after hematopoietic stem cell transplantation is safe without meningococcal vaccination. Biol Blood Marrow Transplant 22:1337-1340

\section{Answers}

1. a; 2. a b; 3. d; 4. a e; 5. c 